\title{
Измерение расстояний до галактик в Местном объеме
}

\author{
А.В. Антипова, Д.И. Макаров, Л.Н. Макарова, И.Д. Караченцев \\ Специальная астрофизическая обсерватория РАН, Нижний Архыз, Россия, 369167 \\ osen.nayti@gmail.com
}

Поступила в редакцию 25 октября 2017 г.

\begin{abstract}
Аннотация. Мы измерили расстояние до 22 галактик методом TRGB по данным космического телескопа "Хаббл". Изображения были получены по программе 13442 "Геометрия и кинематика Местного объема”, в рамках которой снимались наиболее далекие галактики Местного объема. В результате наших измерений в диапазоне 7-8 Мпк пополнение составило $46 \%$ от общего числа галактик, измеренных по вершине ветви красных гигантов (TRGB). В ходе выполнения работы случайно была обнаружена новая карликовая галактика вблизи LV J1157+5638. Две карликовые галактики с абсолютными величинами $M_{V}=-13.26$ и $-9.38 \mathrm{mag}$ образуют физическую пару.

MEASUREMENT OF DISTANCES TO GALAXIES IN THE LOCAL VOLUME, by A. $V$. Antipova, D.I. Makarov, L.N. Makarova, I.D. Karachentsev. We measured distances of 22 galaxies using archive data of the Hubble Space Telescope. Images were obtained in the framework of program 13442 "Geometry and kinematics of the Local Volume". Our measurements increased the number of known TRGB distance in the range from 7 to $8 \mathrm{Mpc}$ on $46 \%$ of the total number of galaxies measured at the tip of the red giant branch (TRGB). During our work we serendipitously discovered a new dwarf galaxy near object LV J1157+5638. Two dwarf galaxies with absolute magnitudes $M_{V}=-13.26$ and -9.38 mag form a physical pair of galaxies.
\end{abstract}

Ключевые слова: близкие галактики, определение расстояний, галактики LV J1157+5638 и LV J1157+5638 sat

\section{1 Введение}

Знание точных расстояний - ключевой момент для изучения физических свойств галактик, их распределения в пространстве и исследования Вселенной в целом. Построение шкалы расстояний чрезвычайно кропотливая работа, требующая высокой точности измерений и учета различных систематических эффектов.

Одним из методов, который позволяет быстро и эффективно оценивать расстояние до галактик вплоть до 10 Мпк, является метод, основанный на светимости вершины ветви красных гигантов. Эффективность метода связана с тем, что используются достаточно яркие звезды $m_{I} \approx-4 \mathrm{mag}$. Он базируется на фотометрии старого звездного населения, что облегчает учет внутреннего поглощения в галактике. Метод применим практически ко всем галактикам, которые разрешаются на звезды, так как старое звездное население встречается в галактиках всех морфологических типов. Метод TRGB не требует больших затрат наблюдательного времени по сравнению с изучением переменных звезд. 
Физический процесс, лежащий в основе метода, хорошо понятен. На последних стадиях жизни маломассивных (1-2 $\left.M_{\odot}\right)$ звезд на ветви красных гигантов образуется вырожденное гелиевое ядро. Снятие вырождения сопровождается термоядерной вспышкой. Из-за наличия вырождения воспламенение гелия происходит при почти постоянной массе ядра. Это, в свою очередь, означает, что вспышка происходит с предсказуемой светимостью. Наблюдательное проявление этой теоретической картины - резкий обрыв функции светимости звезд ветви красных гигантов. Первым, кто предложил использовать ярчайшие красные гиганты для измерения расстояний, был Сэндидж (1971). Он определил абсолютную звездную величину ярчайших красных гигантов как $M_{V}=-3.0 \pm 0.2 \mathrm{mag}$. ДаКоста, Армандрофф (1990) оценили болометрическую величину TRGB как $M_{b o l}=-3.81-0.19[F e / H]$, где $[\mathrm{Fe} / \mathrm{H}]$ - металличность TRGB. Ли и др. (1993) разработали первый количественный метод нахождения обрыва функции светимости RGB звезд, и это дало начало широкому распространению данного метода. Мэндез и др. (2002) опубликовали свой подход, основанный на подгонке функции светимости методом максимального правдоподобия. Улучшенный вариант этого метода в статье Макарова и др. (2006), Рицци и др. (2007) позволил повысить точность оценки модуля расстояния до $0.02 \mathrm{mag}$.

\section{2 Данные}

В 2014 году на космическом телескопе “Хаббл” проводились наблюдения по программе 13442 (PI: Талли) - “Геометрия и кинематика Местного объема”. Основной целью программы было: определение расстояний с точностью до 200 кпк; определение локального поля скоростей в качестве индикатора распределения темной материи; изучение звездного населения в ближайших галактиках.

Данные были получены с камерой ACS в двух фильтрах $(F 814 W, F 606 W)$ для каждой галактики. Большинство галактик были сняты с экспозицией около 1000 секунд в каждом фильтре.

\section{3 Определение расстояния до галактик}

По описанной выше программе мы провели звездную фотометрию 28 галактик с помощью программного пакета Dolphot (Долфин, 2000) с рекомендуемыми параметрами. Для повышения контрастности ветви красных гигантов на диаграмме цвет - звездная величина исключались области активного звездообразования, дефекты матрицы и области вокруг ярких звезд. Положение TRGB определялось с помощью программного пакета TRGBtool, написанного Макаровым и др. (2006) на базе системы MATLAB. Данная программа определяет звездную величину вершины ветви красных гигантов методом максимального правдоподобия, показатель цвета находится как робастная оценка бегущего среднего. На рис. 1 представлен пример галактики ESO308-022 из нашей выборки галактик. Справа располагается изображение, полученное на космическом телескопе “Хаббл”, слева - диаграмма цвет - звездная величина, по которой определялось положение TRGB.

Для определения модуля расстояния использовалась калибровка Рицци и др. (2007). Результаты измерения расстояний приведены в табл. 1 , где Name - имя галактики; $m_{T R G B}$ - звездная величина TRGB в фильтре $F 814 W$ без учета поглощения в галактике и ошибка, с которой она была определена; $c_{T R G B}$ - цвет $(F 606 W-F 814 W)$ TRGB и его ошибка, $(m-M)_{0}$ - модуль расстояния, ошибка, с которой был определен модуль расстояния; Distance - расстояние в Мпк и его ошибка. Для 6 галактик мы не смогли определить расстояние: ESO495-021, ESO496-010, VCC 381, KDG 229, UGC 9660, KKR 8. Эти галактики слишком далекие и не разрешаются на звезды. 

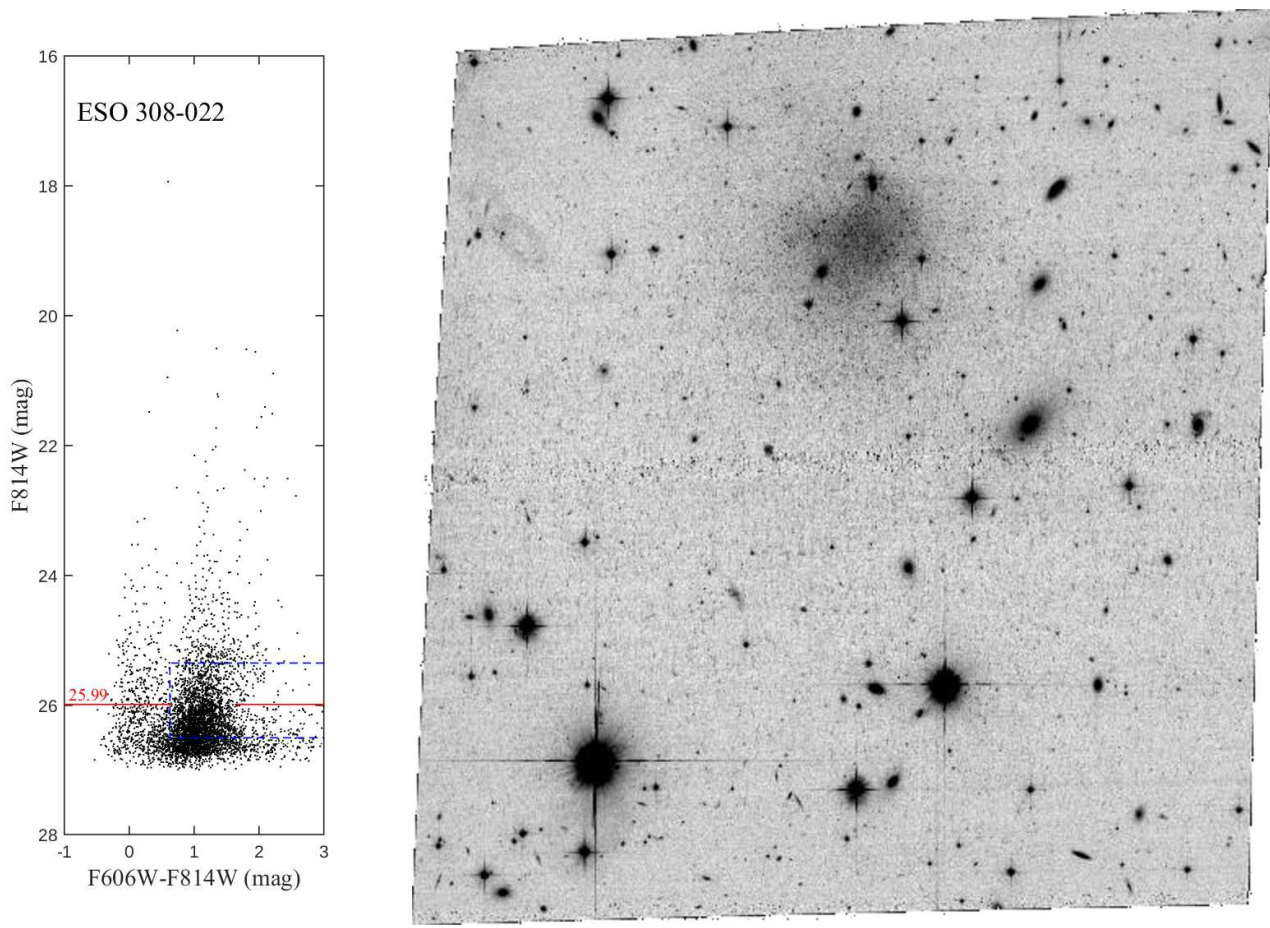

Рис. 1. Диаграмма цвет - звездная величина и изображение галактики ESO308-022, полученное на космическом телескопе “Хаббл”. На диаграмме сплошной горизонтальной линией показано положение TRGB, штриховой линией выделена область, где осуществлялся поиск TRGB

Таблица 1. Результаты фотометрии и измерения расстояния до галактик

\begin{tabular}{lcccc}
\hline Name & $m_{T R G B}$ & $c_{T R G B}$ & $(m-M)_{0}$ & Distance \\
\hline ESO409-015 & $25.80 \pm 0.10$ & $1.12 \pm 0.03$ & $29.85 \pm 0.11$ & $9.3 \pm 0.5$ \\
NGC 59 & $24.59 \pm 0.02$ & $1.247 \pm 0.004$ & $28.61 \pm 0.06$ & $5.27 \pm 0.15$ \\
6dF0335-45 & $25.49 \pm 0.06$ & $1.10 \pm 0.05$ & $29.56 \pm 0.08$ & $8.2 \pm 0.3$ \\
ES0364-029 & $25.91 \pm 0.03$ & $1.08 \pm 0.01$ & $29.91 \pm 0.07$ & $9.6 \pm 0.3$ \\
NGC2188 & $25.87 \pm 0.03$ & $1.24 \pm 0.01$ & $29.88 \pm 0.07$ & $9.47 \pm 0.26$ \\
ESO308-022 & $25.99 \pm 0.06$ & $1.16 \pm 0.02$ & $29.92 \pm 0.08$ & $9.6 \pm 0.4$ \\
FG 202 & $24.89 \pm 0.04$ & $1.06 \pm 0.01$ & $28.80 \pm 0.08$ & $5.76 \pm 0.21$ \\
UGC6757 & $24.27 \pm 0.11$ & $1.04 \pm 0.03$ & $28.33 \pm 0.13$ & $4.6 \pm 0.7$ \\
DDO129 & $24.89 \pm 0.04$ & $1.06 \pm 0.02$ & $28.96 \pm 0.07$ & $6.2 \pm 0.2$ \\
NGC4485 & $25.73 \pm 0.02$ & $1.23 \pm 0.01$ & $29.75 \pm 0.06$ & $8.92 \pm 0.26$ \\
NGC4707 & $25.19 \pm 0.03$ & $1.07 \pm 0.02$ & $29.27 \pm 0.06$ & $7.13 \pm 0.22$ \\
NGC4861 & $25.93 \pm 0.07$ & $1.13 \pm 0.03$ & $29.99 \pm 0.09$ & $10.0 \pm 0.4$ \\
UGC 9992 & $26.25 \pm 0.08$ & $1.16 \pm 0.02$ & $30.27 \pm 0.10$ & $11.3 \pm 0.5$ \\
IC 4710 & $25.43 \pm 0.03$ & $1.27 \pm 0.01$ & $29.35 \pm 0.07$ & $7.40 \pm 0.24$ \\
IC 4870 & $25.84 \pm 0.04$ & $1.27 \pm 0.01$ & $29.71 \pm 0.08$ & $8.8 \pm 0.3$ \\
IC 5201 & $26.17 \pm 0.03$ & $1.14 \pm 0.01$ & $30.23 \pm 0.06$ & $11.1 \pm 0.3$ \\
NGC 7640 & $26.02 \pm 0.05$ & $1.30 \pm 0.01$ & $29.89 \pm 0.08$ & $9.5 \pm 0.3$ \\
AGC111945 & $25.57 \pm 0.07$ & $1.14 \pm 0.02$ & $29.53 \pm 0.09$ & $8.07 \pm 0.33$ \\
KK191 & $25.70 \pm 0.05$ & $1.17 \pm 0.03$ & $29.75 \pm 0.08$ & $8.89 \pm 0.08$ \\
LV2335-37 & $25.43 \pm 0.05$ & $0.97 \pm 0.03$ & $29.51 \pm 0.08$ & $8.0 \pm 0.3$ \\
LV J1157+5638 & $27.74 \pm 0.07$ & $0.97 \pm 0.03$ & $29.82 \pm 0.09$ & $9.22 \pm 0.38$ \\
LV J1157+5638 sat & $25.66 \pm 0.13$ & $1.00 \pm 0.06$ & $29.76 \pm 0.11$ & $8.95 \pm 0.42$ \\
\hline
\end{tabular}




\section{4 Обнаружение компаньона галактики LV J1157+5638}

В процессе работы был найден ранее неизвестный компаньон галактики LV J1157+5638, который мы обозначили как LV J1157+5638 sat. Обе галактики разрешаются на звезды, и мы смогли оценить их расстояние методом TRGB. Галактика LV J1157+5638 находится на расстоянии $9.22 \pm 0.38$ Мпк, а LV J1157+5638 sat на расстоянии $8.95 \pm 0.42$ Мпк. Расстояния обеих галактик одинаковы в пределах ошибок измерения. Проекционное расстояние между LV J1157+5638 и LV J1157+5638 sat составляет 3.9 кпк. На основе этих данных мы можем сделать вывод, что обнаруженная нами галактика является физическим спутником LV J1157+5638.

Для этих двух галактик мы провели также апертурную фотометрию программой АРРНОТ в круговых апертурах. Поток от галактики LV J1157+5638 измерялся в апертурах с общим центром и радиусами, возрастающими от $150\left(7.5^{\prime \prime}\right)$ до $500\left(25^{\prime \prime}\right)$ пикселей с шагом 25 пикселей $\left(1.25^{\prime \prime}\right)$. Уровень фона определялся в двух кольцах радиусами 550 и 600 и ширинами 150 и 300 пикселей соответственно. Разница между этими двумя измерениями приводит к неопределенности интегрального потока галактики в двух фильтрах на уровне 1-2\% от его величины. Вклад в поток от попадания в апертуру 500 пикселей фоновых галактик оценивается как $1.2 \%$ и 1.8 \% от потока исследуемой галактики в фильтрах $F 606 W$ и $F 814 W$ соответственно. Таким образом, для интегральных звездных величин получены оценки $F 606 \mathrm{~W}=16.55 \pm 0.04 \mathrm{mag}, F 814 \mathrm{~W}=16.07 \pm 0.04 \mathrm{mag}$ (в величине ошибок учтены неопределенности, связанные с уровнем фона).

Для измерения потоков от LV J1157+5638 sat использовались апертуры радиусами 60-160 пикселей $\left(3-8^{\prime \prime}\right)$ с шагом 10 пикселей $\left(0.5^{\prime \prime}\right)$. Уровень фона измерялся в двух кольцевых апертурах радиусами и шириной соответственно 200 и 100, 300 и 150 пикселей. Эта галактика значительно слабее предыдущей, что влечет за собой более высокие неопределенности, связанные с вариацией уровня фона, до $5 \%$ в фильтре $F 606 W$. Вклад фоновых объектов и оставшихся после автоматической первичной обработки снимков дефектных пикселей в потоке объекта в апертуре радиусом 160 пикселей оценивается на уровне $6 \%$ в $F 606 W$ и $9 \%$ в $F 814 W$. Оценки интегральных величин галактики равны $F 606 W=20.35 \pm 0.06 \mathrm{mag}, F 814 W=19.77 \pm 0.06 \mathrm{mag}$.

Переход от звездных величин в фильтрах HST к величинам в системе Джонсона-Козинса и коррекция за межзвездное поглощение в нашей Галактике проводились в специализированном пакете Synphot с использованием команды CALCPHOT. Величина поглощения в Галактике составляет $E(B-$ $V)=0.017 \mathrm{mag}$ (Шлегель и др., 1998). Итоговые звездные величины равны $V=16.61 \pm 0.04 \mathrm{mag}$, $I=16.05 \pm 0.04 \mathrm{mag}$ для галактики LV J1157+5638, и $V=20.43 \pm 0.06 \mathrm{mag}, I=19.75 \pm 0.06 \mathrm{mag}$ для LV J1157+5638 sat.

Исходя из полученных результатов мы можем оценить абсолютные звездные величины галактик. Абсолютная звездная величина галактики LV J1157+5638 в фильтре $V$ равна $-13.26 \pm 0.10 \mathrm{mag}$, в фильтре $I$ равна $-13.80 \pm 0.10 \mathrm{mag}$. Для галактики LV J1157+5638 sat абсолютные звездные величины в фильтрах $V$ и $I$ равны соответственно $-9.38 \pm 0.13 \mathrm{mag}$ и $-10.04 \pm 0.13 \mathrm{mag}$.

\section{5 Заключение}

Мы исследовали 28 галактик по 27 полям, изображения которых были получены на космическом телескопе “Хаббл”. Для 6 галактик мы не смогли измерить расстояния, так как они слишком далекие и не разрешаются на звезды, для 22 наши измерения расстояний оказались в диапазоне от 4 до 11.5 Мпк со средней ошибкой расстояния 0.15-0.3 Мпк. Наши измерения увеличили количество галактик с известными TRGB расстояниями на $18 \%$ в диапазоне 6-7 Мпк, на 46 \% для 7-9 Мпк и на $24 \%$ для расстояний 9-10 Мпк.

В ходе выполнения работы была обнаружена новая карликовая галактика с абсолютной звездной величиной в фильтре $V$, равной $-9.38 \pm 0.13 \mathrm{mag}$. Она располагается на проекционном расстоянии 3.9 кпк от карликовой иррегулярной галактики LV J1157+5638. Совпадение в пределах ошибок измерений расстояний для этих двух галактик позволяет сделать вывод о том, что они образуют физическую пару карликовых галактик. $\mathrm{K}$ настоящему времени за пределами Местной группы обнаружен ряд объектов схожей светимости. Один из них, Apples 1, был также обнаружен случайным 


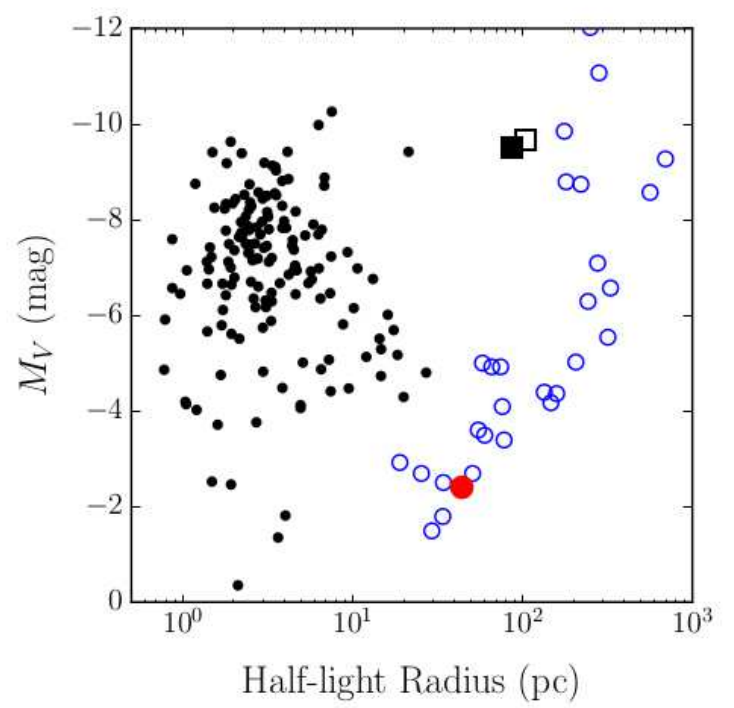

Рис. 2. Положение LV J1157+5636 sat (белый квадрат) отмечено на диаграмме эффективный радиус - абсолютная звездная величина галактики. Положение карликовых галактик (спутников Млечного Пути) показано пустыми кружками, шаровых скоплений Млечного Пути - черными точками, Tucana III - сплошным кружком, Apples 1 - черным квадратом

образом в ходе обзора Apples (Паскуали и др., 2005). Похожие спутники находились в ходе специального обзора вокруг M81 и CenA, но в отличие от работ Чибоукас и др. (2009), Мюллера и др. (2016), мы нашли спутник около карликовой галактики с абсолютной звездной величиной $-13.3 \mathrm{mag}$. На рис. 2 мы сравниваем свойства обнаруженного нами спутника LV J1157+5636 sat с карликовыми галактиками, найденными вокруг Млечного Пути. График взят из статьи Симона и др. (2017), где описывается обнаружение галактики Tucana III. На графике положение шаровых скоплений Млечного пути показано точками, карликовые галактики, открытые вокруг Млечного пути - открытыми кружками, Tucana III - заполненным кружком. Помимо уже имеющихся объектов мы нанесли положение Apples 1 (заполненный квадрат) и LV J1157+5638 sat (открытый квадрат). Как видно из графика, обнаруженный нами объект попадает в область карликовых галактик.

Благодарности. Результаты этой работы основаны на данных космического телескопа "Хаббл" NASA/ESA, полученных из архива Научного института космического телескопа. Эта работа выполнена при поддержке гранта РНФ № 14-12-00965.

\section{Литература}

Да-Коста, Армандрофф (Da Costa G.S., Armandroff T.E.) // Astron. J. 1990. V. 100. P. 162. Долфин (Dolphin A.E.) // Publ. Astron. Soc. Pacific. 2000. V. 112. P. 1397.

Ли и др. (Lee M.G., Freedman W.L., Madore B.F.) // Astrophys. J. 1993. V. 417. P. 553.

Макаров и др. (Makarov D., Makarova L., Rizzi L., et al.) // Astron. J. 2006. V. 132. P. 2729.

Мэндез и др. (Mendez B., Davis M., Moustakas J., et al.) // Astron. J. 2002. V. 124. P. 213.

Мюллер и др. (Müller O., Jerjen H., Pawlowski M.S., et al.) // Astron. Astrophys. 2016. V. 595. id. A119.

Паскуали и др. (Pasquali A., Larsen S., Ferreras I., et al.) // Astron. J. 2005. V. 129. P. 148.

Рицци и др. (Rizzi L., Tully R.B., Makarov D., et al.) // Astrophys. J. 2007. V. 661. P. 815.

Симон и др. (Simon J.D., Li T.S., Drlica-Wagner A., et al.) // Astrophys. J. 2017. V. 838. P. 11. 
Сэндидж (Sandage A.R.) // Proc. Study Week on Nuclei of Galaxies / Ed. O'Connell D.J.K. Amsterdam: North Holland. 1971. P. 601.

Шлегель и др. (Schlegel D.J., Finkbeiner D.P., Davis M.) // Astrophys. J. 1998. V. 500. P. 525.

Чибоукас и др. (Chiboucas K., Karachentsev I.D., Tully R.B.) // Astron. J. 2009. V. 137. P. 3009. 